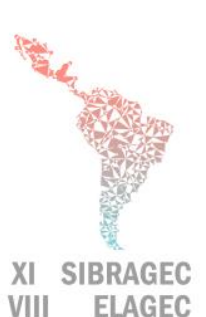

VIII ELAGEC
XI SIMPÓSIO BRASILEIRO DE GESTÃO E ECONOMIA DA CONSTRUÇÃO

VIII ENCUENTRO LATINOAMERICANO DE GESTIÓN Y ECONOMÍA DE LA CONSTRUCCIÓN

Do conhecimento à ação: práticas avançadas de gestão da produção Londrina, Paraná, Brasil. 23 a 25 de Outubro de 2019

\title{
PLANEJAMENTO BASEADO EM LOCAIS EM ATIVIDADES E LOCAIS COM CARACTERÍSTICAS DE NÃO REPETITIVIDADE
}

\author{
SLOBODZIAN, Jocemar (1); GRANJA, Ariovaldo Denis (2); \\ (1) Laboratório de Gerenciamento na Construção (LAGERCON); Departamento de Arquitetura e \\ Construção, Faculdade de Engenharia Civil, Arquitetura e Urbanismo; Universidade Estadual de \\ Campinas, UNICAMP, +55 41 99652-7013, e-mail: js_engcivil@yahoo.com.br (2) Professor Associado, \\ Laboratório de Gerenciamento na Construção (LAGERCON); Departamento de Arquitetura e \\ Construção, Faculdade de Engenharia Civil, Arquitetura e Urbanismo; Universidade Estadual de \\ Campinas, UNICAMP, +55 19 3521-2082, e-mail: adgranja@unicamp.br
}

\begin{abstract}
Construction planning can be classified into two categories, an activity-based (ABP) or a location-based $(L B P)$. The ABP includes traditional planning methods, such as the Critical Path Method (CPM). LBP has many benefits when applied to high repetition constructions. The objective of this paper is to verify whether the procedures of LBP, already consolidated for repetitive constructions, apply in a non-repetitive work structure and what benefits are obtained when compared to ABP. A case study is conducted in a common area of a commercial building in Brazil, which was originally scheduled using ABP planning methods and compared with LBP. The results show that it is possible to apply the procedures related to LBP in nonrepetitive construction, assuring the flow of work and resources.
\end{abstract}

Keywords: Construction, Planning, Location-Based Management System.

\section{INTRODUÇÃ̃O}

Um planejamento ineficaz ainda é uma das principais causas de desvios de prazo em construções (ALENCAR; CÂNDIDO, 2017; FILIPPI; MELHADO, 2015; MUIANGA; GRANJA; RUIZ, 2015). Essa afirmação é um indicativo de que o planejamento de obras ainda requer atenção e aprimoramento. Um detalhamento maior no planejamento pode auxiliar na implantação de algumas técnicas as quais aumentariam a eficiência da produção (BULHÕES; FORMOSO; AVELLAN, 2003). Pesquisas anteriores sugerem duas categorias para planejamento de obras: i) Planejamento Baseado em Atividades (PBA) que inclui técnicas como o Critical Path Method (CPM) e ii) o Planejamento Baseado em Locais (PBL) que incluí técnicas como a Linha de Balanço (LDB) e o Location Based Management System (LBMS) (KENLEY; SEPPÄNEN, 2009). É muito comum na literatura encontrar relatos de que o PBL é mais adequado para obras com um alto grau de repetição quando comparado ao PBA (ANGELIM; HEINECK, 2010), pois esse promove um maior fluxo de recursos (OLIVIERI; GRANJA; PICCHI, 2016).

Por outro lado, a aplicação de PBL em obras com atividades ou locais não repetitivos ainda é escassa na literatura. Linnik, Berghede e Ballard (2013) fazem uso de PBL para uma obra não repetitiva e afirmam que é viável e vantajoso, entretanto, recomendam

SLOBODZIAN, J.; GRANJA, A.D. Planejamento baseado em locais em atividades e locais com características de não repetitividade. In: SIMPÓSIO BRASILEIRO DE GESTÃO E ECONOMIA DA CONSTRUÇÃO, 11., 2019, Londrina. Anais [...]. Porto Alegre: ANTAC, 2019. Disponível em: https://www.antaceventos.net.br/index.php/sibragec/sibragec2019/paper/view/469 
futuras pesquisas para avaliar o impacto nas durações e produtividade. Valente et al (2014) desenvolveram diretrizes para o uso de LDB nas locais sem repetitividade, e afirmam que o plano traz mais transparência para equipe, melhora o controle e diminui a alocação de recursos. Obras não repetitivas precisam necessitam um melhor detalhamento do trabalho pela dificuldade das equipes estimarem a velocidade de produção (TOMMELEIN, 2017). O LBMS é uma abordagem que ajuda a remover essa complexidade de obras não repetitivas, entretanto ainda existem questões que necessitam de respostas para o avanço do conhecimento científico nesta temática, tais como: i) qual é a melhor maneira de dividir os locais de trabalho em situações de não repetitividade e ii) como levar em consideração a interdependência entre os locais, alocação, fluxo e variabilidade dos recursos (MURGUIA; URBINA, 2018). O uso do PBL, no contexto de obras com locais e atividades não repetitivas, se constitui em uma lacuna no conhecimento, a qual esse artigo almeja ajudar a preenchê-la.

Esse artigo é parte de uma dissertação de mestrado, ainda em desenvolvimento, que tem a intenção de determinar os benefícios da utilização do PBL em obras não repetitivas. Neste artigo, o objetivo é verificar se os procedimentos do PBL também se aplicam em uma obra não repetitiva e quais benefícios são obtidos quando comparados com o PBA.

\section{REVISÃO DE LITERATURA}

O PBA é também conhecido como planejamento tradicional (OLIVIERI; GRANJA; PICCHI, 2016). Técnicas como CPM são amplamente disseminados em meio acadêmico e profissional, e são peças fundamentais do Gerenciamento de Projetos (HOWELL; KOSKELA, 2000; PMI, 2017). A popularidade desses métodos deve-se em parte pelo desenvolvimento de softwares com uma interface amigável como Primavera ${ }^{\circledR} \mathrm{e}$ Microsoft Project $^{\circledR}$ (BALDWIN; BORDOLI, 2015). Essa abordagem de planejamento resulta em um gráfico de Gantt, onde as atividades são listadas no eixo vertical e dispostas em uma barra ao longo da escala de tempo do eixo horizontal (O'BRIEN; PLOTNICK, 2015). Os procedimentos para elaboração de um PBA estão apresentados no Quadro 1.

\section{Quadro 1 - PBA}

\begin{tabular}{|l|l|}
\hline \multicolumn{1}{|c|}{ Procedimento } & \multicolumn{1}{c|}{ Descrição } \\
\hline $\begin{array}{l}\text { Estrutura analítica do projeto } \\
\text { (EAP) }\end{array}$ & Decomposição do escopo da construção em pacotes de trabalho. \\
\hline Definir atividades & $\begin{array}{l}\text { Identificação das ações necessárias para produzir as entregas de } \\
\text { cada pacote. }\end{array}$ \\
\hline Estimar durações & Critério selecionado pelo planejador. \\
\hline Sequenciamento & Atribuição de vínculos entre atividades e cálculo das folgas. \\
\hline Recursos & Atribuição, verificação da disponibilidade e nivelamento. \\
\hline Otimização e Riscos & $\begin{array}{l}\text { Aplicação de técnicas de compressão para redução de prazo e } \\
\text { atribuição de buffers para combater incertezas. }\end{array}$ \\
\hline Linha de base & Criação e validação de cronograma \\
\hline
\end{tabular}

Fonte: adaptado de O'BRIEN; PLOTNICK (2015)

Por sua vez, o diagrama produzido no PBL apresenta os locais no eixo vertical e uma escala de tempo no eixo horizontal. As tarefas são representadas por linhas que demonstram a evolução da mesma e mostram onde cada equipe trabalha e em que 
momento. A inclinação da linha significa o ritmo em que a tarefa é executada (KENLEY; SEPPÄNEN, 2010). Os procedimentos para elaboração de um PBL estão apresentados no Quadro 2.

\section{Quadro 2 - PBL}

\begin{tabular}{|l|l|}
\hline \multicolumn{1}{|c|}{ Procedimento } & \multicolumn{1}{c|}{ Descrição } \\
\hline $\begin{array}{l}\text { Estrutura de locais do projeto } \\
\text { (ELP) }\end{array}$ & Decomposição da obra em locais com complexidades similares. \\
\hline Quantidades & Atribuir a quantidade de trabalho em cada local. \\
\hline Construção de tarefas & $\begin{array}{l}\text { Reunião de um conjunto de atividades que se repetem em } \\
\text { múltiplos locais ou possam ser executadas por uma única } \\
\text { equipe. }\end{array}$ \\
\hline Tamanho ótimo de equipe & Definição da taxa de produção, jornada e tamanho das equipes. \\
\hline Sequenciamento & $\begin{array}{l}\text { No LBMS, a sequência execução é arranjada por meio de cinco } \\
\text { layers que vinculam tarefas e locais entre si. }\end{array}$ \\
\hline Otimização e Riscos & $\begin{array}{l}\text { Ajuste do ritmo ou sequência de produção, quebra de } \\
\text { continuidade ou divisão de tarefas onde necessário. }\end{array}$ \\
\hline Linha de base & Criação e validação de cronograma \\
\hline
\end{tabular}

Fonte: adaptado de (KENLEY; SEPPANEN, 2010)

PBA e PBL possuem muitas diferenças, as quais fogem do escopo desta pesquisa. Para este artigo, entretanto, alguns pontos precisam ser destacados. No PBA, o foco está nas durações das atividades, as quais são obtidas geralmente com base na experiência do planejador e contém quantidade expressiva de buffers. No PBL, o foco está no fluxo das equipes pelos diferentes locais e no ritmo de produção obtido por parâmetros de produção bem definidos (REZAEI, 2015).

\section{MÉTODO DE PESQUISA}

Um estudo de caso foi utilizado para verificar a aplicação dos procedimentos do PBL indicados no Quadro 2. A obra analisada é a execução da "periferia" de um edifício comercial. A quantidade de trabalho e o tipo de acabamento são diferentes de local para local, além disso, não são todos os serviços que se repetem em todos os locais. Essas complexidades adicionais caracterizam locais e atividades não repetitivas. A construtora em questão disponibilizou informações da obra bem como um cronograma de barras elaborado em Microsoft Project ${ }^{\circledR}$ (PBA/CPM), realizado por um planejador experiente. Este cronograma trata-se da versão inicial do planejamento de uma obra já concluída portanto, a participação do pesquisador teve uma característica passiva.

Os dados coletados foram sintetizados e cada tarefa do diagrama de barras foi atribuído a um local. A conversão do formato de apresentação foi realizada para que o cronograma original (PBA) possa ser comparado posteriormente com o PBL. Esse processo resultou no diagrama da Figura 1, o qual originalmente era apresentado em várias folhas A1 e agora em uma única folha A4. Essa Figura contém, portanto, a síntese dos dados originais de um PBA elaborado com base nos processos do Quadro 1, porém com uma apresentação gráfica típica de um PBL. 


\section{SIBRAGEC - ELAGEC 2019 - de 23 a 25 de Outubro - LONDRINA - PR}

O cronograma do PBL apresentado na Figura 2 foi obtido realizando as etapas sugeridas por Kenley e Seppanen (2010) que estão resumidas no Quadro 2. As quantidades foram coletadas dos projetos e as taxas de produção de acordo com dados da própria construtora. O sequenciamento procurou inicialmente promover o máximo possível de fluxo ininterrupto com uma equipe única. O cronograma foi otimizado com ajustes nos ritmos, na sequência de locais de produção, e, em alguns casos, foi necessário quebrar continuidade ou dividir tarefas em mais de um local. A otimização do cronograma do PBL foi realizada até que se atingisse o mesmo prazo final do cronograma original, para que, dessa forma, ambos pudessem ser comparados em condições semelhantes.

\section{RESULTADOS}

A aplicação do método resultou nos diagramas das Figuras 1 e 2, os quais representam o PBA e o PBL respectivamente. A leitura de ambos os diagramas é intuitiva, em um eixo estão os locais, no outro eixo o tempo e as linhas inclinadas representam as tarefas. Algumas tarefas foram destacadas em ambos os cronogramas para facilitar as interpretações: amarelo - reboco, azul - forro, vermelho - pintura, verde - limpeza e as demais permaneceram na cor preta. Após uma análise comparativa entre ambos é possível destacar:

- O PBA original possuí originalmente cinco folhas A1 e foi possível reduzi-lo a um diagrama que poderia ser impresso em uma única folha A4 ao utilizar o PBL;

- A quantidade de tarefas que são realizadas no mesmo local ao mesmo tempo reduziu de 89 no PBA para 29 no PBL, ou seja, uma redução de $67 \%$ das inferências. Essas interferências não são explícitas em gráfico como o de Gantt, o que trouxe uma maior transparência ao planejamento;

- A quantidade de equipes distribuídas em vários locais ao mesmo tempo também diminuiu. Por exemplo, a tarefa de reboco (amarelo) no PBA está ocorrendo em alguns períodos de tempo em seis locais ao mesmo tempo. No PBL, o reboco ocorre em apenas dois locais ao mesmo tempo com duas equipes paralelas em uma sequência de trabalho bem definida;

- No PBL, as tarefas evoluem de forma contínua. Por exemplo, na equipe de reboco (amarelo) uma equipe inicia no térreo e permanece ali até concluir e outra inicia no segundo subsolo e avança até concluir o primeiro subsolo. O exemplo da limpeza (verde) é ainda mais claro, a tarefa avança continuamente do primeiro local até o último sem precisar de desmobilização como precisou no PBA;

- Um filtro pode ser aplicado no diagrama para transmitir as informações para a equipe executora, contendo apenas as informações necessárias. A equipe consegue ter uma melhor visualização da sequência de trabalho e obtém-se assim uma melhoria na comunicação;

- No PBA, o segundo subsolo foi concluído bem antes. Isso é causado pela prática comum de planejadores determinarem o início "assim que possível" em cronogramas CPM. No PBL, prioriza-se o fluxo ininterrupto das equipes, mesmo que isso signifique postergar o início da tarefa em alguns setores. A construção enxuta preconiza que equipes não devem aguardar locais serem liberados e locais vazios não devem esperar equipes. Por esse motivo, o PBL não obteve, neste caso, a aplicação dos princípios da construção enxuta de uma forma literal. Obter fluxo ininterrupto trouxe consigo o trade-off de locais aguardando equipe. 
SIBRAGEC - ELAGEC 2019 - de 23 a 25 de Outubro - LONDRINA - PR

- Os benefícios desse replanejamento podem ser questionados como um efeito dado simplesmente por uma visão de um planejador diferente do original. Entretanto, o PBL traz transparência na visualização dos fluxos de equipes pelos locais, o que é muito difícil no PBA, já que esse segundo é baseado na EAP e o primeiro tem a ELP como peça fundamental.

Figura 1 - PBA no formato típico de um PBL

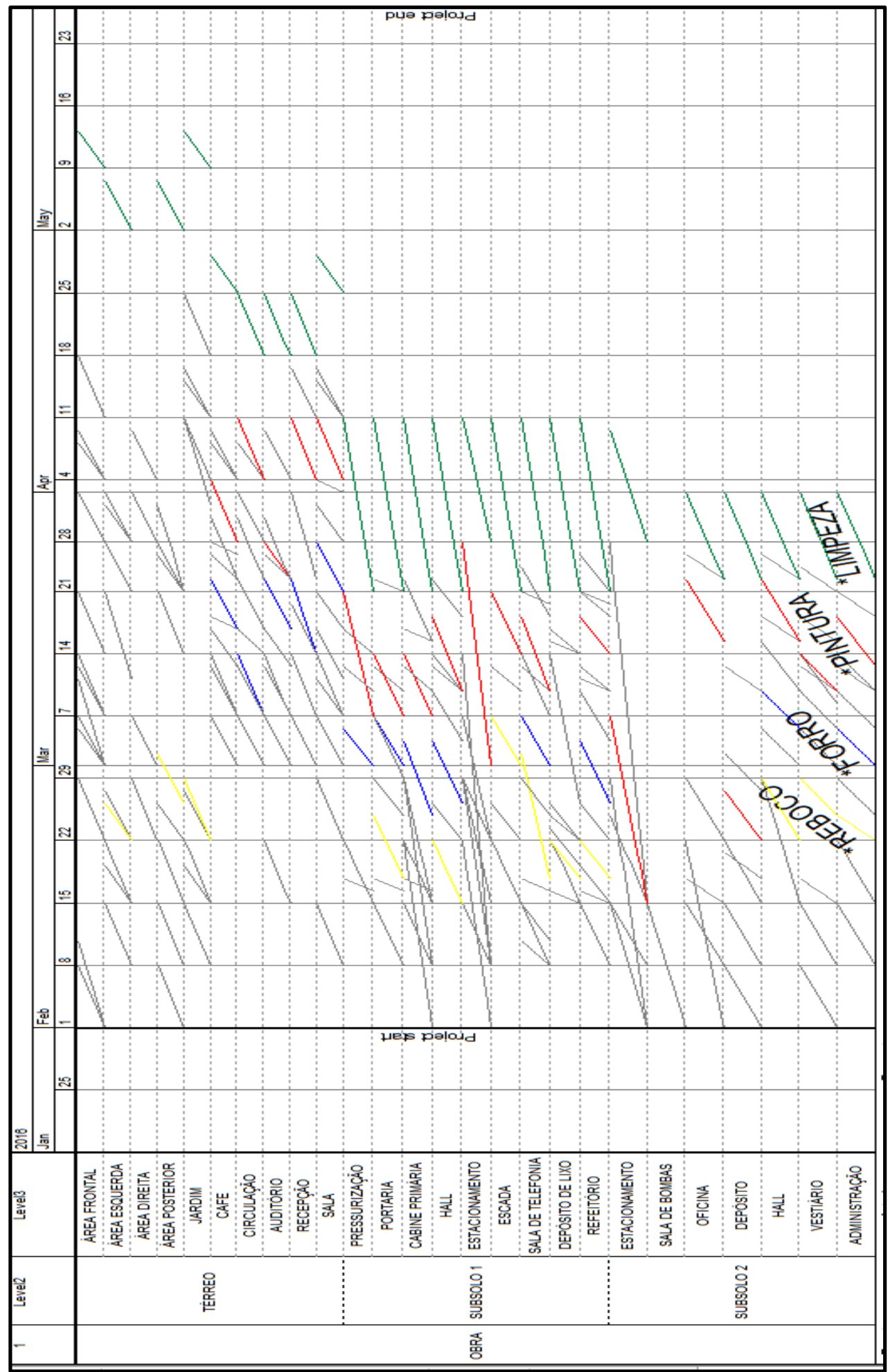

Fonte: $\mathrm{O}$ autor 
SIBRAGEC - ELAGEC 2019 - de 23 a 25 de Outubro - LONDRINA - PR

Figura 2 - PBL

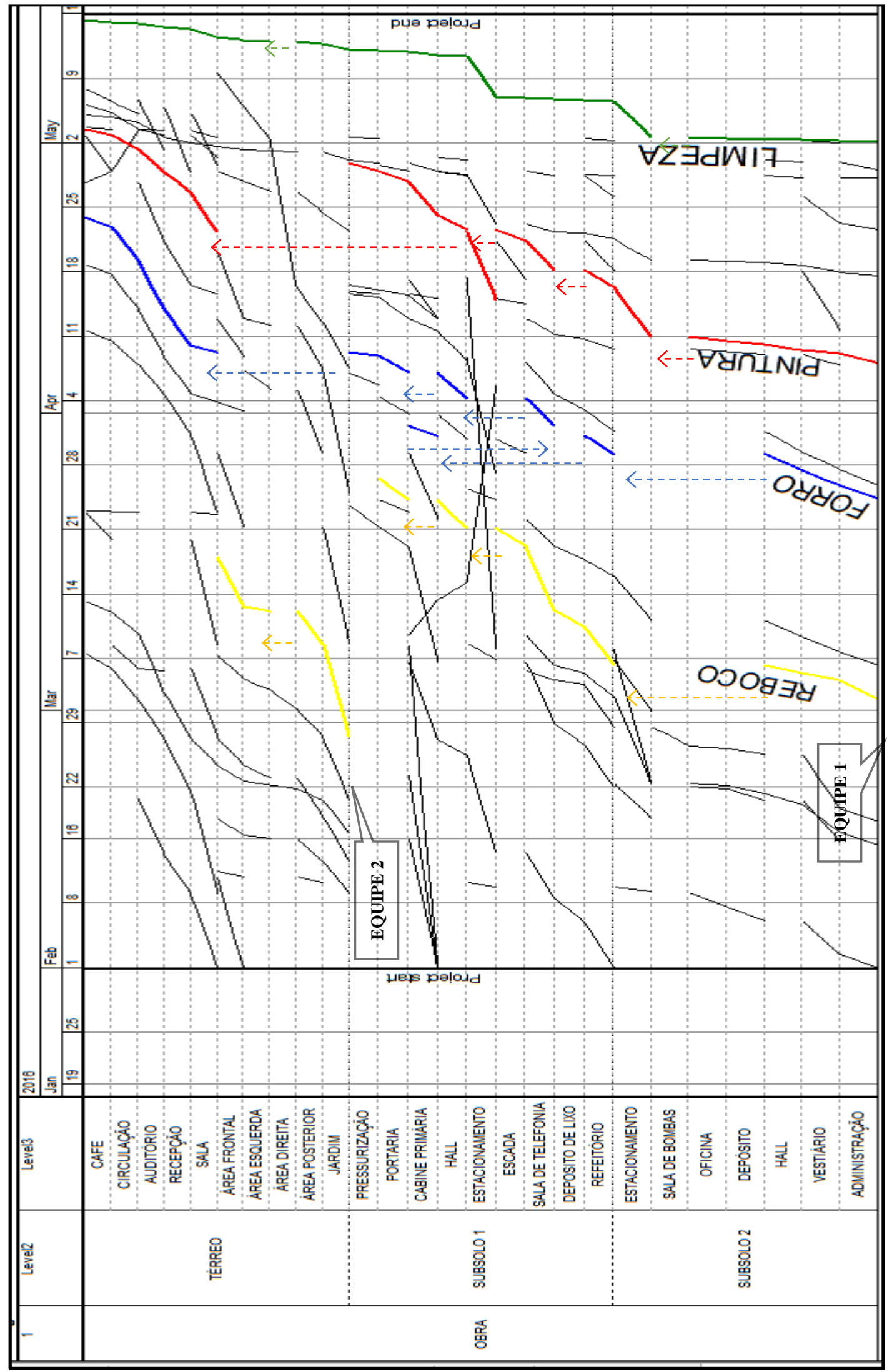

Fonte: $\mathrm{O}$ autor 
SIBRAGEC - ELAGEC 2019 - de 23 a 25 de Outubro - LONDRINA - PR

\section{CONCLUSÕES}

Esse artigo verificou que os procedimentos do PBL, já consolidados em obras repetitivas, são possíveis de serem aplicados também em obras não repetitivas. $\mathrm{O}$ estudo de caso permitiu a constatação de alguns benefícios da utilização do PBL quando comparado ao PBA como o fluxo ininterrupto e a redução de interferências de tarefas executadas em um mesmo local ao mesmo tempo. Com essas conclusões será possível dar segmento na pesquisa de mestrado, onde o PBL poderá ser posto à prova em circunstâncias de não repetitividade ainda mais complexas.

\section{REFERÊNCIAS}

ALENCAR, G. C. DE M.; CÂNDIDO, L. F. Análise do Planejamento e Controle da Produção em uma microempresa construtora. In: VII CONGRESSO BRASILEIRO DE ENGENHARIA DE PRODUÇÃO. Anais... Ponta Grossa, 2017.

ANGELIM, V. L.; HEINECK, L. F. M. Implantação da ferramenta Linha de Balanço em uma obra industrial. In: XIII ENCONTRO NACIONAL DE TECNOLOGIA DO AMBIENTE CONSTRUÍDO. Anais... Canela, 2010.

BALDWIN, A.; BORDOLI, D. Handbook for Construction Planning and Scheduling. Wiley Blackwell, UK, 2015.

BULHÕES, I. R.; FORMOSO, C. T.; AVELLAN, T. V. Gestão dos fluxos físicos e sua integração com o planejamento e controle da produção: Caso de uma empresa de Salvador-BA. In: III SIMPÓSIO BRASILEIRO DE GESTÃ̃ E ECONOMIA DA CONSTRUÇÃO. Anais... São Carlos, 2003.

FILIPPI, G. A. D.; MELHADO, S. B. Um estudo sobre as causas de atrasos de obras de empreendimentos imobiliários na região Metropolitana de São Paulo. Ambiente Construído, v. 15, n. 3, p. 161-173, Porto Alegre, 2015.

HOWELL, G. A.; KOSKELA, L. Reforming Project Management: The Role of Lean Construction. In: 8TH ANNUAL CONFERENCE OF THE INTERNACIONAL GROUP FOR LEAN CONSTRUCTION. Proceedings... Brigthon, UK 2000.

KENLEY, R.; SEPPÄNEN, O. Location-based management of construction projects: Part of a new typology for project scheduling methodologies. WINTER SIMULATION CONFERENCE. Proceedings... Austin USA, 2009.

KENLEY, R.; SEPPANEN, O. Location-based management for construction: Planning, scheduling and control. 1. ed. Abingdon: Spon Press, New York USA, 2010.

LINNIK, M.; BERGHEDE, K.; BALLARD, G. An Experiment in Takt Time Planning Applied to Non-Repetitive Work. In: 21TH ANNUAL CONFERENCE OF INTERNATIONAL GROUP FOR LEAN CONSTRUCTION. Proceedings... Fortaleza, Brazil, 2013.

MUIANGA, E. A. D.; GRANJA, A. D.; RUIZ, J. DE A. Desvios de custos e prazos em empreendimentos da construção civil: categorização e fatores de influência. Ambiente Construído, v. 15, n. 1, p. 79-97, Porto Alegre, 2015.

MURGUIA, D.; URBINA, A. Complex Production Systems: Non-Linear and Non-Repetitive Projects. In: 26TH ANNUAL CONFERENCE OF THE INTERNATIONAL GROUP FOR LEAN CONSTRUCTION. Proceedings... Chennai, India: 2018.

O'BRIEN, J. J.; PLOTNICK, F. L. CPM in Construction Management. 8. ed. McGraw-Hill Education, 2015. 
SIBRAGEC - ELAGEC 2019 - de 23 a 25 de Outubro - LONDRINA - PR

OLIVIERI, H.; GRANJA, A. D.; PICCHI, F. A. Planejamento tradicional, Location-Based Management System e Last Planner System: um modelo integrado. Ambiente Construído, v. 16, n. 1, p. 265-283, Porto Alegre, 2016.

PMI, P. M. I. Um Guia do Conhecimento em Gerenciamento de Projetos (Guia PMBOK). 6. ed. Newton Square, PA, EUA, 2017.

REZAEI, A. Location Based Scheduling In The Form Of Flow Line and Its Comparison to Cpm/Bar Chart Scheduling. International Journal of Eletronics, p. 13, 2015.

TOMMELEIN, I. D. Collaborative Takt Time Planning of Non-Repetitive Work. In: 25TH ANNUAL CONFERENCE OF INTERNATIONAL GROUP FOR LEAN CONSTRUCTION. Proceedings... Heraklion, Greece, 2017.

VALENTE, C. P. et al. Guidelines for Developing a Line of Balance for Non-Repetitive Areas (Common Areas) at a Vertical Residential Building. In: 22ND ANNUAL CONFERENCE OF INTERNATIONAL GROUP FOR LEAN CONSTRUCTION. Proceedings... Oslo, Norway, 2014. 